\title{
Modeling of the near field plume of a Hall thruster
}

\author{
lain D. Boyd ${ }^{\text {a) }}$ and John T. Yim \\ Department of Aerospace Engineering, University of Michigan, Ann Arbor, Michigan 48109-2140
}

(Received 18 November 2003; accepted 30 January 2004)

\begin{abstract}
In this study, a detailed numerical model is developed to simulate the xenon plasma near-field plume from a Hall thruster. The model uses a detailed fluid model to describe the electrons and a particle-based kinetic approach is used to model the heavy xenon ions and atoms. The detailed model is applied to compute the near field plume of a small, $200 \mathrm{~W}$ Hall thruster. Results from the detailed model are compared with the standard modeling approach that employs the Boltzmann model. The usefulness of the model detailed is assessed through direct comparisons with a number of different measured data sets. The comparisons illustrate that the detailed model accurately predicts a number of features of the measured data not captured by the simpler Boltzmann approach. (c) 2004 American Institute of Physics. [DOI: 10.1063/1.1688444]
\end{abstract}

\section{INTRODUCTION}

Hall thrusters represent an efficient form of plasma electric propulsion for spacecraft. In general, electric propulsion is replacing chemical propulsion for many on-board propulsion tasks due to their higher specific impulse. The superior performance provides longer missions and lighter propulsion systems that allows heavier payloads. Continued advances in photovoltaic technology mean that the solar-generated power available on spacecraft is growing. Thus, while the original Hall thrusters presently flying in space on Russian spacecraft are rated at power levels of about $1.5 \mathrm{~kW}$, and current generation Hall thrusters will operate at $5 \mathrm{~kW}$, future devices will be able to operate at considerably higher levels such as $50 \mathrm{~kW}$ and above. The development of electric propulsion systems for high power sources is taking two distinct paths. In one case, single, monolithic thrusters are being investigated. The second approach involves clustering many smaller thrusters.

Modeling of the plasma flows of Hall thrusters is an important activity in two main ways. First, models are being developed to help understand the complex plasma processes inside the thruster with the aim of improving propulsion performance and extending thruster lifetime. Second, models have been developed of the plasma plume from the thruster in order to help assess spacecraft integration issues. The high energy ions created by the thruster can sputter spacecraft surfaces upon impact leading to possible damage and subsequent re-deposition.

The near field plume of a Hall thruster is a physically complex and important region. The relatively high plasma density in the near field makes it accessible to a variety of experimental diagnostic techniques from probes to nonintrusive optical methods such as laser induced fluorescence (LIF). Such data are much more difficult to obtain either inside the thruster or in the plume far field. Therefore, data obtained in the near-field often provide important glimpses

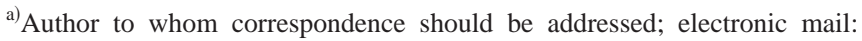
electronic mail: iainboyd@umich.edu
}

of thruster and plume far field processes. In addition, in the development of clusters of Hall thrusters, any interactions between the plumes of the individual thrusters will be strongest in the near field region.

In the present study, the near field plume of a single 200 W class Hall thruster is modeled using a detailed particlefluid hybrid approach. The new aspect of the model involves treating the electrons using a detailed fluid model constructed from the fundamental conservation equations for number, momentum, and energy. The additional physics makes it possible to include the effects of the external cathode in the simulation as well as more accurate simulation of the variation in electron temperature and plasma potential. Details of the model are first presented and compared with the standard approach for simulating Hall thruster plumes. General flow field results from these models are compared. Detailed comparisons of the model results with several different sets of experimental measurements is then made. Conclusions about the usefulness of the detailed model are presented.

\section{HALL THRUSTER AND EXPERIMENTS}

The device considered in the present study is the BHT200 Hall thruster manufactured by Busek, Co. The thruster is operated at $200 \mathrm{~W}$ with a nominal thruster level of $13 \mathrm{mN}$. It is being considered as a component in a cluster of thrusters.

The BHT-200 thruster has been investigated experimentally using both intrusive probes and nonintrusive optical diagnostics. Faraday probes were used by Hargus and Reed ${ }^{1}$ and by $\mathrm{Beal}^{2}$ to measure angular profiles of ion current density in the plume far field. The study in Ref. 1 was performed at a fixed distance of $60 \mathrm{~cm}$ from the thruster. The vacuum chamber is located at the Air Force Research Laboratory, Edwards Air Force Base and is $3.0 \mathrm{~m}$ in length and $1.8 \mathrm{~m}$ in diameter with a pumping speed of $32000 \mathrm{l} / \mathrm{s}$ on xenon resulting in a back pressure of $6.1 \times 10^{-6}$ Torr. The data in Ref. 2 were taken at $50 \mathrm{~cm}$ from the thruster in a $9 \mathrm{~m}$ by $6 \mathrm{~m}$ tank at the University of Michigan. The total pumping speed employed in this facility for these experiments was 140000 
$1 / \mathrm{s}$ on xenon resulting in a back pressure of 1.1 $\times 10^{-6}$ Torr. The thruster was operated at identical conditions in each case. Beal and Gallimore ${ }^{3}$ also used a retarding potential analyzer (RPA) to measure the ion energy distribution function in the plume far field. In addition, a floating emissive probe and a triple Langmuir probe were used to measure the plasma potential, the electron temperature, and the electron density in the near-field plume of the thruster. ${ }^{4}$ Hargus and Reed ${ }^{5}$ used LIF to measure axial and radial velocity components of singly charged xenon ions in the near field of the thruster. Results obtained using the simulation models presented in Sec. III will later be compared with all of these sets of experimental measurements.

\section{MODEL DESCRIPTION}

Hall thrusters primarily use xenon as propellant. The xenon plasma plume is composed of beam ions with velocities on the order of $16 \mathrm{~km} / \mathrm{s}$, low energy charge exchange ions, neutral atoms, and electrons. The total number density is on the order of $10^{18} \mathrm{~m}^{-3}$ that places the plasma in the rarefied flow regime. Computational analysis of Hall thruster plumes is regularly performed using a hybrid particle-fluid formulation. The direct simulation Monte Carlo (DSMC) method $^{6}$ models the collisions of the heavy particles (ions and atoms). The particle in cell (PIC) method ${ }^{7}$ models the transport of the ions in electric fields. Overall, a hybrid approach is employed in which the electrons are modeled using a fluid description. In the present study, we modify an existing axially symmetric PIC-DSMC code developed specifically for modeling the xenon plume from a hollow cathode. ${ }^{8}$ The model provides a detailed treatment of the electron fluid as described below.

\section{A. Plasma dynamics}

Models of Hall thruster plumes have been reviewed by Boyd. ${ }^{9}$ The most successful of these are based on a hybrid approach in which heavy species are modeled using particles and the electrons modeled as a fluid. This is the approach adopted in the present study. Almost all previous hybrid models reduce the electron fluid model to the Boltzmann relation. This requires that the electrons be collisionless, currentless, isothermal, and unmagnetized. All of these assumptions are questionable in a Hall thruster plume, particularly in the plume near field. Despite the simplicity of the model, these hybrid methods have been quite successful in simulating the far-field properties of a number of different Hall thrusters.

As mentioned earlier, the ions and neutrals are treated using a combination of the PIC method ${ }^{7}$ for transporting the ions in electrostatic fields, and the DSMC method ${ }^{6}$ for performing collisions and transporting the neutral atoms. Momentum transfer and charge exchange collisions are the only collision mechanisms implemented.

For the fluid electrons, two different approaches are considered in the present study. The first of these is the Boltzmann model. In this approach, quasineutrality is assumed, which allows the ion density to represent the electron density. By further assuming that the electrons are isothermal, collisionless, and unmagnetized, and that their pressure obeys the ideal gas law, $p_{e}=n_{e} k T_{e}$, the Boltzmann relation is obtained from the electron momentum equation

$$
\phi-\phi^{*}=\frac{k T_{e}}{e} \ln \left(\frac{n_{e}}{n^{*}}\right),
$$

where $n_{e}$ is the electron number density, ${ }^{*}$ indicates a reference state, $\phi$ is the plasma potential, $k$ is Boltzmann's constant, $T_{e}$ is the constant electron temperature, and $e$ is the electron charge. The potential is then differentiated spatially to obtain the electric fields.

The second fluid electron model (termed the Detailed model) involves a much more detailed approach employing all three conservation equations. The electron continuity equation is ${ }^{10}$

$$
\frac{\partial}{\partial t}\left(n_{e}\right)+\nabla \cdot\left(n_{e} \mathbf{v}_{\mathbf{e}}\right)=n_{e} n_{a} C_{i}
$$

where $n_{e}$ is the electron number density, $\mathbf{v}_{\mathbf{e}}$ is the electron velocity vector, $n_{a}$ is the atomic number density, and $C_{i}$ is the ionization rate coefficient. Assuming steady flow, this equation is transformed into a Poisson equation by introducing

$$
n_{e} \mathbf{v}_{\mathbf{e}}=\nabla \psi
$$

such that

$$
\nabla^{2} \psi=n_{e} n_{a} C_{i}
$$

for which numerical solutions are obtained using the standard alternating direction implicit (ADI) method. The spatial distribution of the ion particles gives the electron number density, $n_{e}$, under the assumption of charge neutrality. This allows the electron velocity vector to be determined through solution of Eq. (4). The xenon ionization rate coefficient is expressed as a function of electron temperature using a simple relation proposed by Ahedo et al. ${ }^{11}$

$$
C_{i}=\sigma_{i} c_{e}\left(1+\frac{T_{e} \varepsilon_{i}}{\left(T_{e}+\varepsilon_{i}\right)^{2}}\right) \exp \left(-\frac{\varepsilon_{i}}{T_{e}}\right),
$$

where $\sigma_{i}=5 \times 10^{-20} \mathrm{~m}^{2}$ is a reference cross section, $c_{e}$ is the mean thermal speed of electrons, $\varepsilon_{i}$ is the ionization energy of xenon $(12.7 \mathrm{eV})$, and the electron temperature $T_{e}$ is in electron volts. Use of this thermal ionization coefficient assumes that the electron stream velocity always lies below the threshold level for direct ionization of xenon (about 2 $\times 10^{6} \mathrm{~m} / \mathrm{s}$ ) which is true for all flow conditions considered.

The electron momentum equation is given by ${ }^{10}$

$$
\frac{\partial}{\partial t}\left(m_{e} n_{e} \mathbf{v}_{\mathbf{e}}\right)+m_{e} n_{e}\left(\mathbf{v}_{\mathbf{e}} \cdot \boldsymbol{\nabla}\right) \mathbf{v}_{\mathbf{e}}=-e n_{e} \mathbf{E}-\nabla p_{e}+\mathbf{R},
$$

where $m_{e}$ is the mass of an electron, $\mathbf{E}$ is the electric field, $p_{e}$ is the electron pressure, and $\mathbf{R}$ is the friction term. It is further assumed that the electrons behave as a perfect gas $\left(p_{e}\right.$ $=n_{e} k T_{e}$ ), and that the friction term is given by

$$
\mathbf{R}=\frac{e n_{e} \mathbf{j}}{\sigma},
$$


where $\mathbf{j}$ is the current density and $\sigma$ is the electrical conductivity.

Assuming a steady state, neglecting the inertial term on the left hand side of Eq. (6), and introducing the plasma potential $-\boldsymbol{\nabla} \phi=\mathbf{E}$, a generalized Ohm's law is obtained:

$$
\mathbf{j}=\sigma\left[-\boldsymbol{\nabla} \phi+\frac{1}{e n_{e}} \boldsymbol{\nabla}\left(n_{e} k T_{e}\right)\right]
$$

For given $n_{e}, \mathbf{v}_{\mathrm{e}}$, and $T_{e}$, the charge continuity condition

$$
\boldsymbol{\nabla} . \mathbf{j}=0
$$

is then solved to obtain the plasma potential. This equation is written as a Laplace equation with weak source terms and is again solved using an ADI scheme.

The electron energy equation is given by ${ }^{10}$

$$
\begin{aligned}
& \frac{\partial}{\partial t}\left(\frac{3}{2} n_{e} k T_{e}\right)+\frac{3}{2} n_{e}\left(\mathbf{v}_{\mathbf{e}} \cdot \boldsymbol{\nabla}\right) k T_{e}+p_{e} \boldsymbol{\nabla} \cdot \mathbf{v}_{\mathbf{e}} \\
& \quad=\nabla \cdot \kappa_{e} \nabla T_{e}+\mathbf{j} \cdot \mathbf{E} 3 \frac{m_{e}}{m_{i}} \nu_{e} n_{e} k\left(T_{e}-T_{H}\right)-n_{e} n_{a} C_{i} \varepsilon_{i},
\end{aligned}
$$

where $m_{i}$ is the ion mass, $\nu_{e}$ is the total electron collision frequency, $\kappa_{e}$ is the electron thermal conductivity, and $T_{H}$ is the heavy particle temperature. Again assuming a steady state, and dividing by the thermal conductivity

$$
\begin{aligned}
\nabla^{2} T_{e}= & -\nabla \ln \left(\kappa_{e}\right) \cdot \nabla T_{e}+\frac{1}{\kappa_{e}}\left(-\mathbf{j} \cdot \mathbf{E}+\frac{3}{2} n_{e}\left(\mathbf{v}_{\mathbf{e}} \cdot \nabla\right) k T_{e}\right. \\
& \left.+p_{e} \boldsymbol{\nabla} \cdot \mathbf{v}_{\mathbf{e}}+3 \frac{m_{e}}{m_{i}} \nu_{e} n_{e} k\left(T_{e}-T_{H}\right)+n_{e} n_{a} C_{i} \varepsilon_{i}\right),
\end{aligned}
$$

where $\mathbf{j}$ is obtained from Eq. (8) after the plasma potential is calculated. Equation (11) is again a Laplace equation with weak source terms that is solved using the ADI approach. Effects of ionization are included in the simulation to capture any regions of significant xenon ion production and it is therefore appropriate to include the associated effects in the electron energy equation. However, the computations subsequently showed no significant effect of ionization on the electron energy and this is also found to be true for excitation loss terms. The latter are therefore omitted from the modeling.

Finally, the electron transport coefficients are evaluated using their basic definitions ${ }^{10}$

$$
\begin{aligned}
& \sigma=\frac{e^{2} n_{e}}{m_{e} \nu_{e}}, \\
& \kappa_{e}=\frac{2.4}{1+\frac{\nu_{\mathrm{ei}}}{\sqrt{2} \nu_{e}} m_{e} n_{e} T_{e}},
\end{aligned}
$$

where $\nu_{e}=\nu_{\mathrm{ei}}+\nu_{\mathrm{en}}, \nu_{\mathrm{ei}}$ is the ion-electron collision frequency, $\nu_{\mathrm{en}}$ is the neutral-electron collision frequency, and these frequencies are evaluated for the xenon system using cross sections provided in Ref. 10. Note that, for each time step, the numerical scheme iterates several times through the solution of Eqs. (9) and (10) due to the coupling between $\phi$ and $T_{e}$.

\section{B. Collision dynamics}

The DSMC method uses particles to simulate collision effects in rarefied gas flows by collecting groups of particles into cells which have sizes of the order of a mean free path. Pairs of these particles are then selected at random and a collision probability is evaluated that is proportional to the product of the relative velocity and collision cross section for each pair. The probability is compared with a random number to determine if that collision occurs. If so, some form of collision dynamics is performed to alter the properties of the colliding particles.

There are two basic classes of collisions that are important in the Hall thruster plumes: (1) elastic (momentum exchange) and (2) charge exchange. Elastic collisions involve only exchange of momentum between the participating particles. For the systems of interest here, this may involve atom-atom or atom-ion collisions. For atom-atom collisions, the variable hard sphere ${ }^{6}$ collision model is employed. For xenon, the collision cross section is

$$
\sigma_{\mathrm{EL}}(\mathrm{Xe}, \mathrm{Xe})=\frac{2.12 \times 10^{-18}}{g^{2 \omega}} \mathrm{m}^{2},
$$

where $g$ is the relative velocity and $\omega=0.12$ is related to the viscosity temperature exponent for xenon. For atom-ion elastic interactions, the following cross section of Dalgarno et al. ${ }^{12}$ is employed:

$$
\sigma_{\mathrm{EL}}\left(\mathrm{Xe}, \mathrm{Xe}^{+}\right)=\frac{6.42 \times 10^{-16}}{g} \mathrm{~m}^{2} .
$$

The model of Ref. 12 predicts that the elastic cross section for interaction between an atom and a doubly charged ion is twice that for an atom and a singly charged ion. It should be noted that the model of Ref. 11 employs a polarization potential and therefore is only valid for low energy (a few eV) collisions. In all elastic interactions, the collision dynamics is modeled using isotropic scattering together with conservation of linear momentum and energy to determine the postcollision velocities of the colliding particles. ${ }^{6}$

Charge exchange concerns the transfer of one or more electrons between an atom and an ion. For singly charged ions, the following cross section measured by Pullins et al. ${ }^{13}$ and Miller $e t$ al. ${ }^{14}$ is used:

$$
\begin{aligned}
\sigma_{\mathrm{CEX}}\left(\mathrm{Xe}, \mathrm{Xe}^{+}\right)= & {\left[-23.30 \log _{10}(g)+142.21\right] \times 0.8423 } \\
& \times 10^{-20} \mathrm{~m}^{2} .
\end{aligned}
$$

Also reported in Refs. 13 and 14 are charge exchange cross sections for the interaction where a doubly charged ion captures two electrons from an atom. These cross sections are less than a factor of 2 lower than the values for the singly charged ions at corresponding energies. In the present model, it is assumed that there is no transfer of momentum accompanying the transfer of the electron(s). This assumption is based on the premise that charge exchange interactions are primarily at long range. 


\section{Boundary conditions}

For the computations of the Hall thruster plumes, boundary conditions must be specified at several locations: (1) at the thruster exit; (2) at the cathode exit; (3) along the outer edges of the computational domain; and (4) along all solid surfaces in the computational domain.

Several macroscopic properties of the plasma exiting the thruster are required for the computations. Specifically, the plasma potential, the electron temperature, and for each of the particle species we require the number density, velocity, and temperature. In the real device, these properties vary radially across the exit plane. The approach to determining these properties involves a mixture of analysis and estimation. The basic performance parameters of mass flow rate, thrust, and total ion current are assumed to be known. The neutrals are assumed to exit the thrust at the sonic speed corresponding to some assumed value for their temperature. Finally, divergence angles for the lower $\left(\theta^{-}\right)$and upper $\left(\theta^{+}\right)$edges of the exit channel must be assumed. Combining all this information then allows all species densities and the ion velocities to be determined. Determination of the properties of multiple charge states, for example $\mathrm{Xe}^{2+}$ is considered in the present study, requires knowledge of the current fraction of that state.

In the detailed model, the external cathode of the hall thruster can be modeled. While the actual cathode provides essentially a point source of electrons that therefore involves a three dimensional flow, in the present study it is modeled within the axially symmetric framework of the code. This is not a bad assumption given the high mobility of the electrons that rapidly forms a symmetric flow field. The boundary conditions required for the electrons at the exit of the cathode are the plasma potential, the electron temperature, and the electron current.

Both fluid and particle boundary conditions are required at the outer edges of the computational domain. The usual field conditions employed simply set the electric fields normal to the boundary edges equal to zero. Similarly the gradients in electron temperature normal to the surfaces of the outer boundaries are set to zero. The particle boundary condition is to simply remove from the computation any particle crossing the domain edge. The finite back pressure (the values are listed in Sec. II) of each of the two facilities considered in this study is included by simulating a fixed density background of xenon atoms at room temperature. These atoms can collide with heavy species emitted by the thruster and so can make an important contribution to the charge exchange plasma. In addition, the background atoms affect the neutral-electron collision frequency $\nu_{\text {en }}$ that appears in the transport coefficients of the electron fluid model.

The solid wall surfaces of the Hall thruster are also included in the computation. Along these walls, the plasma potential is set to zero and zero gradient in electron temperature is employed. Any ions colliding with the walls are neutralized. Both atoms and neutralized ions are scattered back into the flow field from the surface of the thruster wall assuming diffuse reflection at a temperature of $300 \mathrm{~K}$.
TABLE I. Thruster exit conditions assumed in the two models.

\begin{tabular}{lccccccc}
\hline \hline Model & $\begin{array}{c}T_{e} \\
(\mathrm{eV})\end{array}$ & $\begin{array}{c}T_{i} \\
(\mathrm{eV})\end{array}$ & $\begin{array}{c}T_{n} \\
(\mathrm{~K})\end{array}$ & $\begin{array}{c}U_{i} \\
(\mathrm{~km} / \mathrm{s})\end{array}$ & $\begin{array}{c}\theta^{+} \\
(\mathrm{deg})\end{array}$ & $\begin{array}{c}\theta^{-} \\
(\mathrm{deg})\end{array}$ & $\begin{array}{c}\phi \\
(\mathrm{V})\end{array}$ \\
\hline Boltzmann & 2 & 2 & 750 & 17.3 & 30 & -30 & 20 \\
Detailed & 6 & 2 & 750 & 13.3 & 20 & 0 & 90 \\
\hline \hline
\end{tabular}

\section{RESULTS}

The operating conditions of the BHT-200 Hall thruster considered in the present study are as follows: ${ }^{1-5}$ flow rate $=9.5 \mathrm{sccm}$, discharge current $=0.85 \mathrm{~A}(85 \%$ is attributed to ions of which $18 \%$ by current fraction is attributed to $\mathrm{Xe}^{2+}$, the $15 \%$ current due to electrons is included in the detailed model $)$, voltage $=240 \mathrm{~V}$, and specific impulse $=1300 \mathrm{~s}$. As discussed above, the thruster operating conditions are converted into boundary conditions for the plume code at the thruster exit. To obtain good agreement between the simulations and the data measured in the far field plume, different sets of thruster exit conditions are required for the Boltzmann and Detailed models. The values employed are listed in Table I and will be discussed later. The simulations are performed on a computational mesh of 133 by 118 nonuniform, rectangular cells that is extended to a distance of about $60 \mathrm{~cm}$ from the thruster. Typically, 300000 particles are employed and the simulations require about 2 and $10 \mathrm{~h}$ of CPU time for the Boltzmann and Detailed models, respectively.

\section{A. Flow field results}

An overall impression of the flow field is provided in Figs. 1 and 2 showing the plasma potential for the entire flow field obtained with the Boltzmann and Detailed models, respectively. These plots demonstrate the very significant difference in the overall flow fields generated by the two different fluid electron models. The Boltzmann model gives relatively weak gradients in plasma potential and therefore in

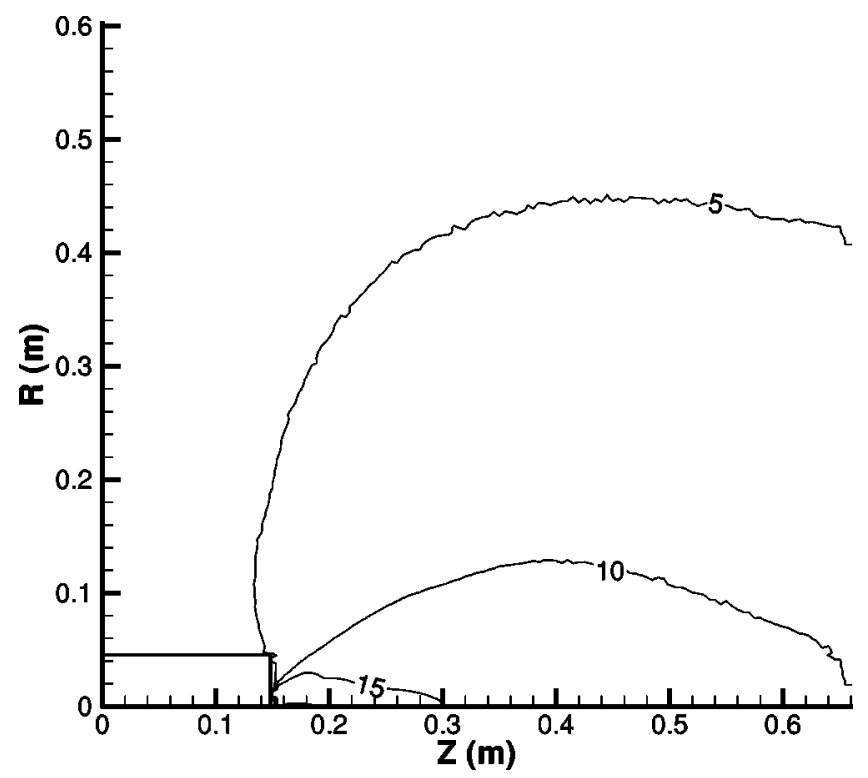

FIG. 1. Contours of plasma potential (in V) for the plume of the BHT-200 computed using the Boltzmann model. 


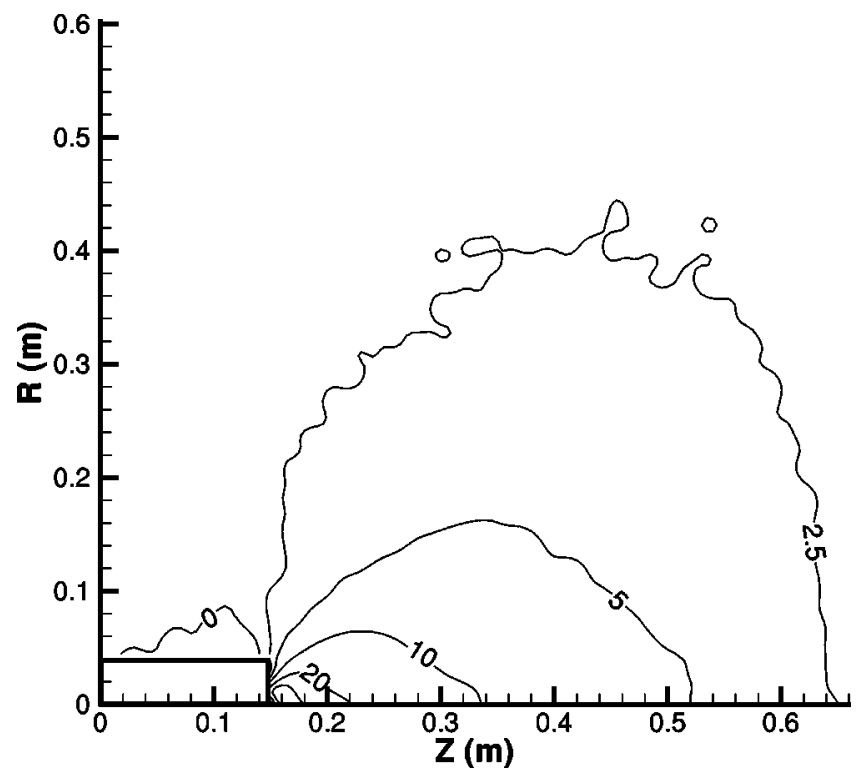

FIG. 2. Contours of plasma potential (in V) for the plume of the BHT-200 computed using the Detailed model.

electric field with a total variation in potential only slightly greater than $10 \mathrm{~V}$. By comparison, the potential gradients, and electric fields, associated with the Detailed model are much stronger, with an overall variation in potential of about $90 \mathrm{~V}$. Hence, it is expected that the Detailed model will produce significantly greater levels of ion acceleration.

\section{B. Far field results}

Assessment of the plume models is first performed in the far field by making direct comparisons of the simulation results with a number of different experimentally measured data sets reported in Refs. 1-5. In Figs. 3 and 4, comparisons are provided for angular profiles of ion current density at a distance of $50 \mathrm{~cm}$ from the thruster (the data from Ref. 1 were obtained at $60 \mathrm{~cm}$ and have been scaled to $50 \mathrm{~cm}$ based

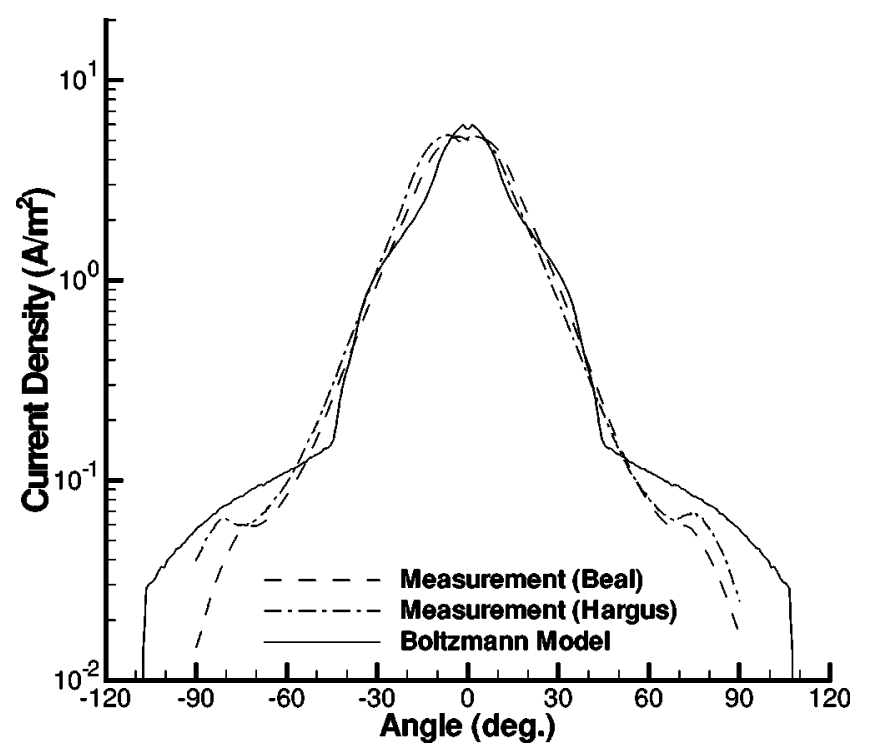

FIG. 3. Angular profiles of ion current density at $50 \mathrm{~cm}$ from the thruster.

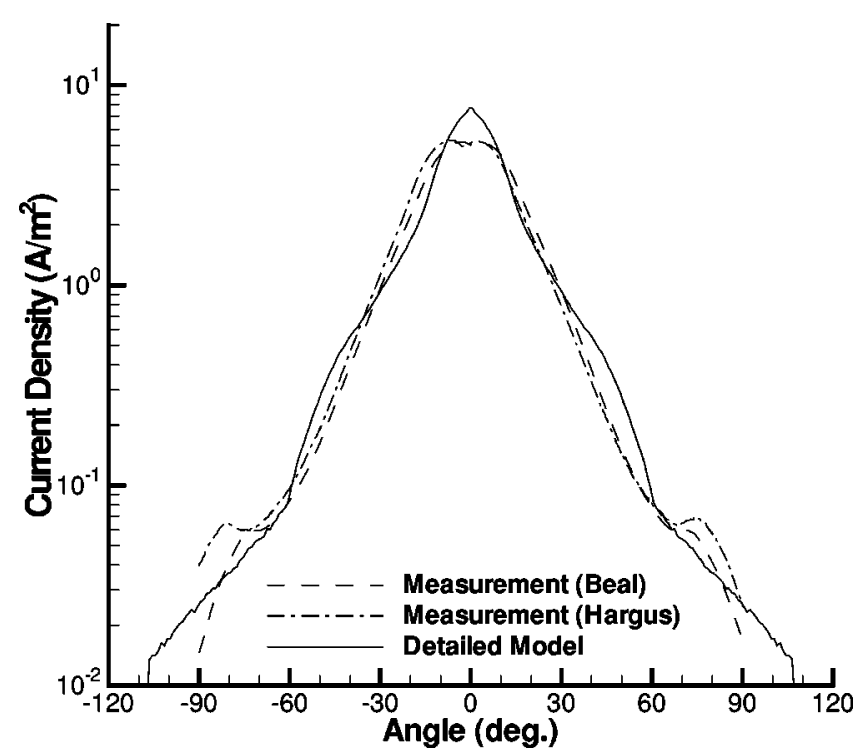

FIG. 4. Angular profiles of ion current density at $50 \mathrm{~cm}$ from the thruster.

on the square of the distance from the thruster). The two sets of measured data were obtained using a Faraday cup., ${ }^{1,2}$ Clearly, excellent agreement is obtained between the two sets of measured data. In Fig. 3, the Boltzmann model provides a higher peak current density on axis, but generally gives good agreement with the measured profiles. This is the best level of agreement that could be obtained based on variation of the thruster exit conditions listed in Table I. The profile obtained with the Detailed model shown in Fig. 4 is also the best result obtained through variation of the thruster exit conditions. This profile offers even better agreement with the measured data.

Figures 5 and 6 make comparisons of the ion energy distribution function measured on the plume axis at a distance of $50 \mathrm{~cm}$ from the thruster using the RPA. ${ }^{3}$ Both simulations provide excellent agreement with the measured data. Note that the computed ion energy distribution function in

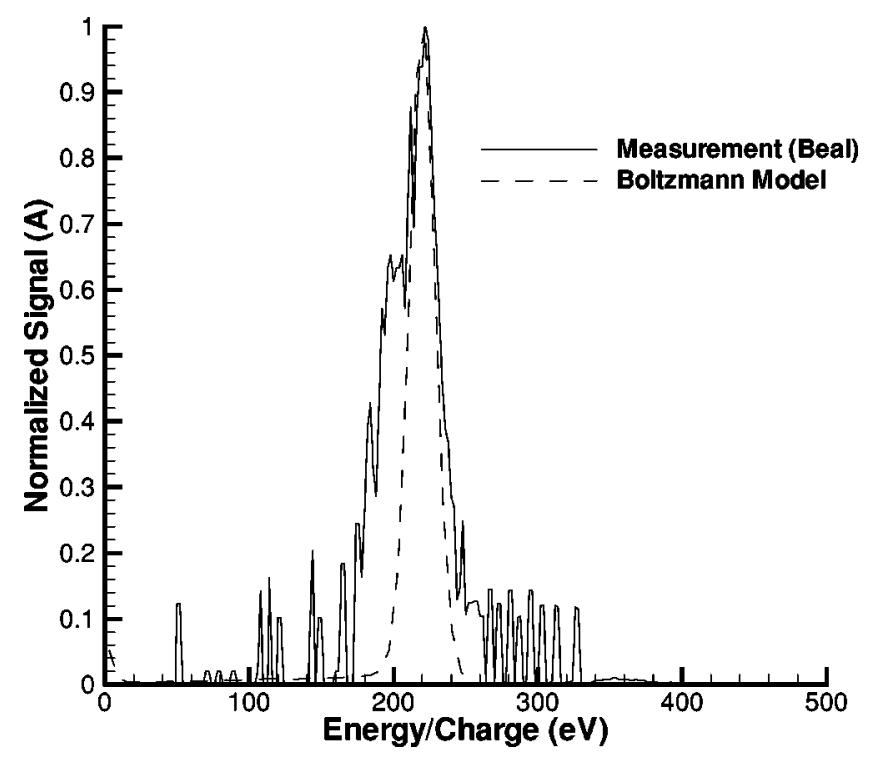

FIG. 5. Ion energy distribution function on axis at $50 \mathrm{~cm}$ from the thruster. 


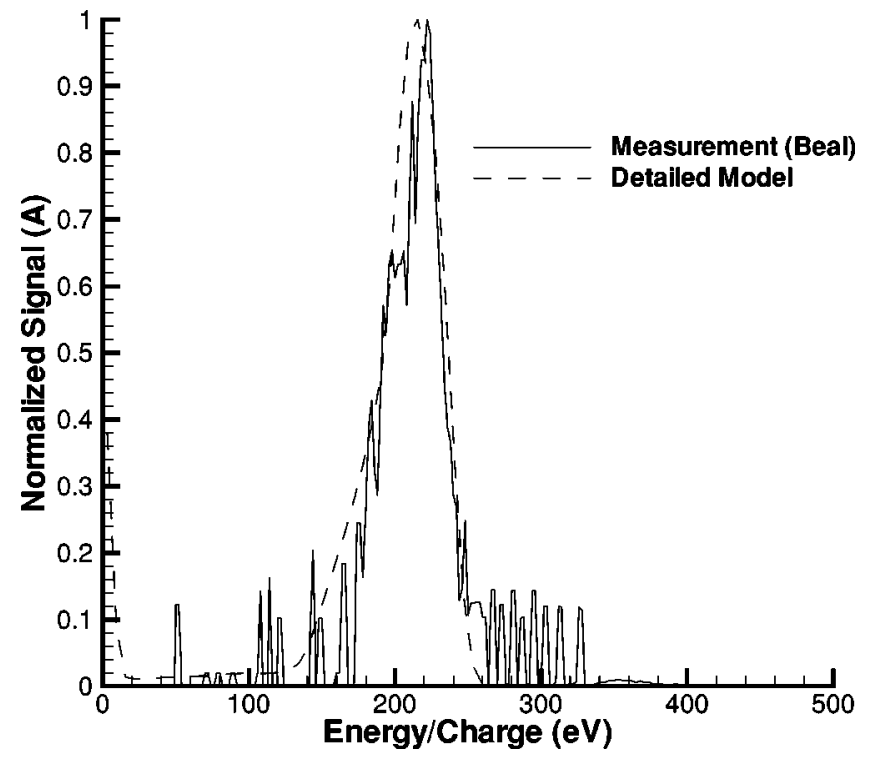

FIG. 6. Ion energy distribution function on axis at $50 \mathrm{~cm}$ from the thruster.

this location is most sensitive to the ion velocity assumed at the thruster exit. The width of the distribution obtained with the Boltzmann model could be increased closer to the measured data by increasing the ion temperature at the thruster exit. In the Detailed model, the exit ion velocity of $13.3 \mathrm{~km} / \mathrm{s}$ is consistent with the measured specific impulse for the thruster of about 1300 s. By comparison, the Boltzmann model can only give agreement with the measured energy distribution by assuming an ion velocity of $17.7 \mathrm{~km} / \mathrm{s}$ at the thruster exit, which is inconsistent with the specific impulse. It will also be shown later that LIF measurements indicate that the ion velocity at the thruster exit is close to $13 \mathrm{~km} / \mathrm{s}$. Thus, at least for this particular thruster, the Boltzmann model only gives good agreement with the far field ion energy distribution function by assuming an erroneously high thruster exit ion velocity. This occurs because the Boltzmann model fails to produce sufficient ion acceleration in the near field of the plume. As discussed with reference to Figs. 1 and 2 , the Detailed model predicts a strong degree of ion acceleration in this region and rapidly accelerates the ions from their thruster exit velocity of $13.3 \mathrm{~km} / \mathrm{s}$ to a value of about 18 $\mathrm{km} / \mathrm{s}$ that corresponds to the peak of the measured energy distribution function. This represents a clear advantage of the Detailed model over the Boltzmann model for simulating Hall thruster plumes.

\section{Near field results}

Figure 7 shows a comparison for plasma potential along the plume centerline. The measured data are obtained using a floating emissive probe. ${ }^{4}$ The Detailed model shows significant decrease in potential and offers excellent agreement with the measured data. By comparison, the Boltzmann model indicates a very small change in potential. Figures 8 and 9 show radial profiles of plasma potential at an axial distance of $50 \mathrm{~mm}$ from the thruster obtained with the Boltzmann and Detailed models, respectively. The measurements were taken on two different BHT-200 thrusters arranged in a

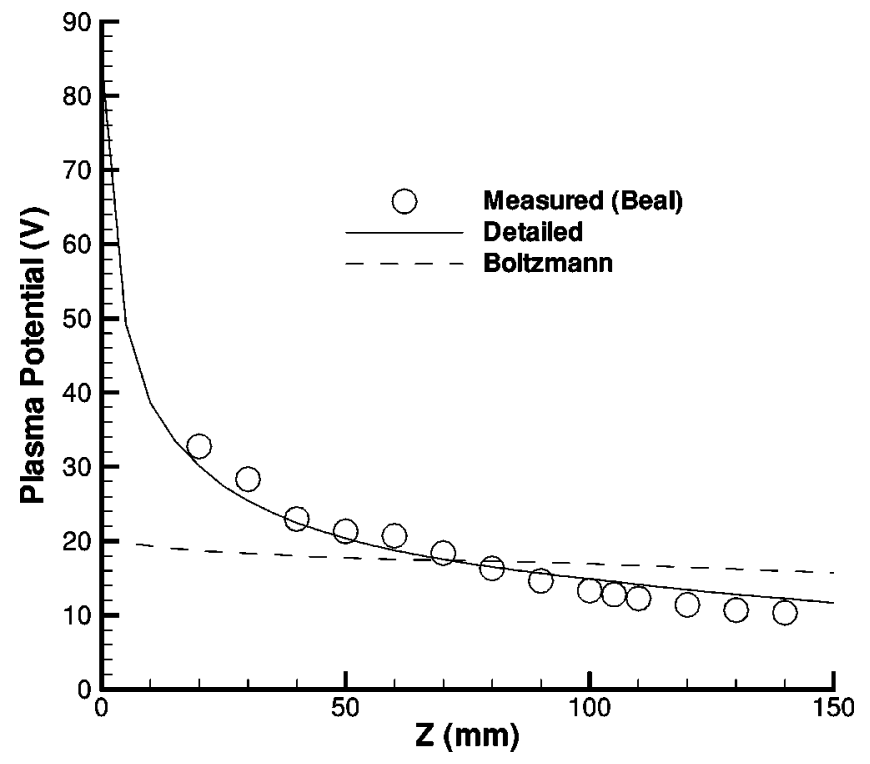

FIG. 7. Axial profiles of plasma potential along the plume centerline.

cluster of four devices, fired one at a time. Both sets of measurements are included in these plots to indicate the levels of repeatability of the experiments (which is generally very good). The simulation results for each thruster are obtained from a single computation and are therefore identical and just shifted along the radial axis for plotting purposes. Figure 8 shows that by appropriate selection of the thruster exit potential, the Boltzmann model can reproduce the peaks of the measured profiles. However, the radial decay of potential is under predicted. The Detailed model provides better prediction of the radial gradients, but still over predicts the measured data. Similar radial profiles are shown in Figs. 10 and 11 at a distance of $100 \mathrm{~mm}$ from the thruster. The profiles from the Boltzmann model are almost identical to those

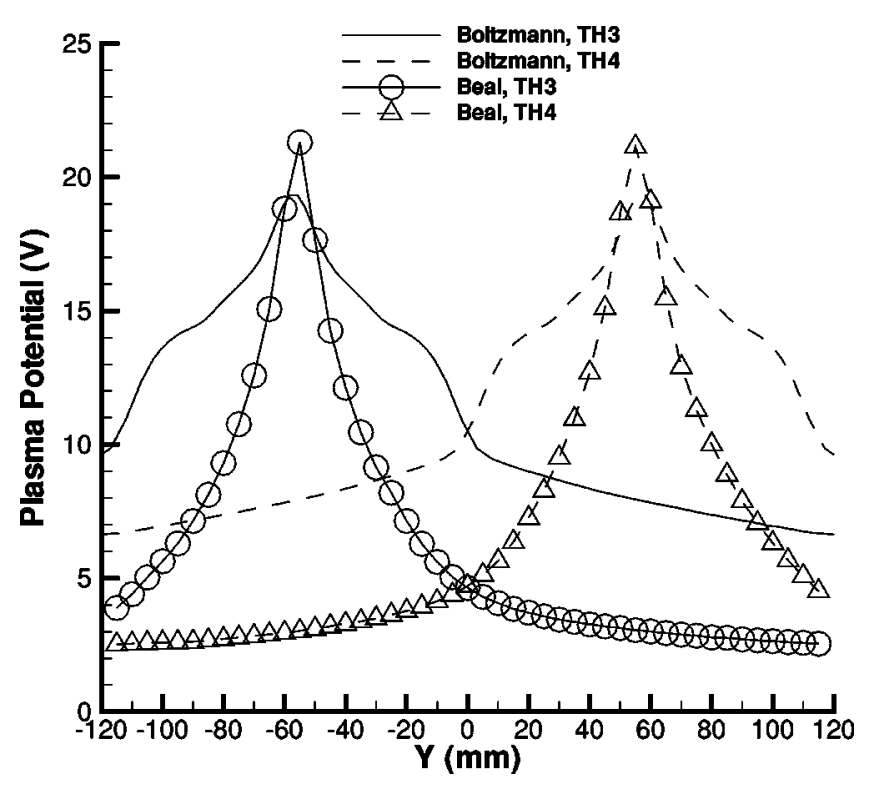

FIG. 8. Radial profiles of plasma potential at an axial distance of $50 \mathrm{~mm}$ from the thruster. 


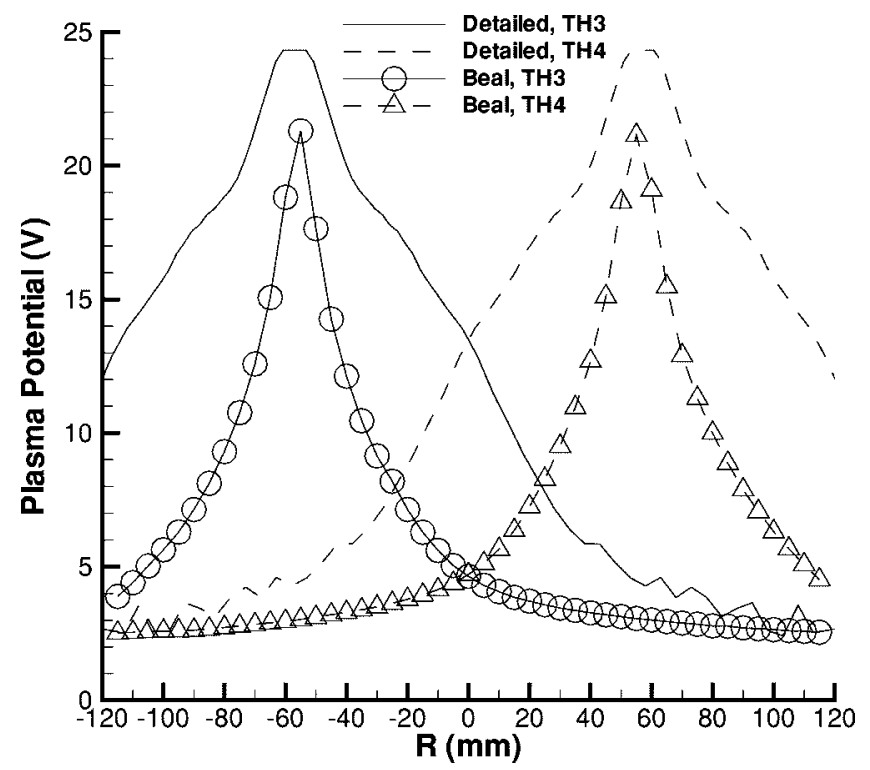

FIG. 9. Radial profiles of plasma potential at an axial distance of $50 \mathrm{~mm}$ from the thruster.

in Fig. 8 and overpredict the potential significantly. The profiles from the Detailed model provide much better agreement with the measured data.

A triple Langmuir probe was also used in Ref. 4 to measure the electron temperature and number density in the near field region. The variation in electron temperature along the plume centerline is shown in Fig. 12. Once again, the Detailed model provides good agreement with the measured variation, whereas the Boltzmann model assumes a constant value of $2 \mathrm{eV}$. Figures 13, 14, and 15 show radial profiles of electron temperature at distances of 50,150, and $250 \mathrm{~mm}$ from the thruster, respectively. Since the Boltzmann results are all flat lines at $2 \mathrm{eV}$, only the Detailed model results are provided. In general, although the Detailed model provides

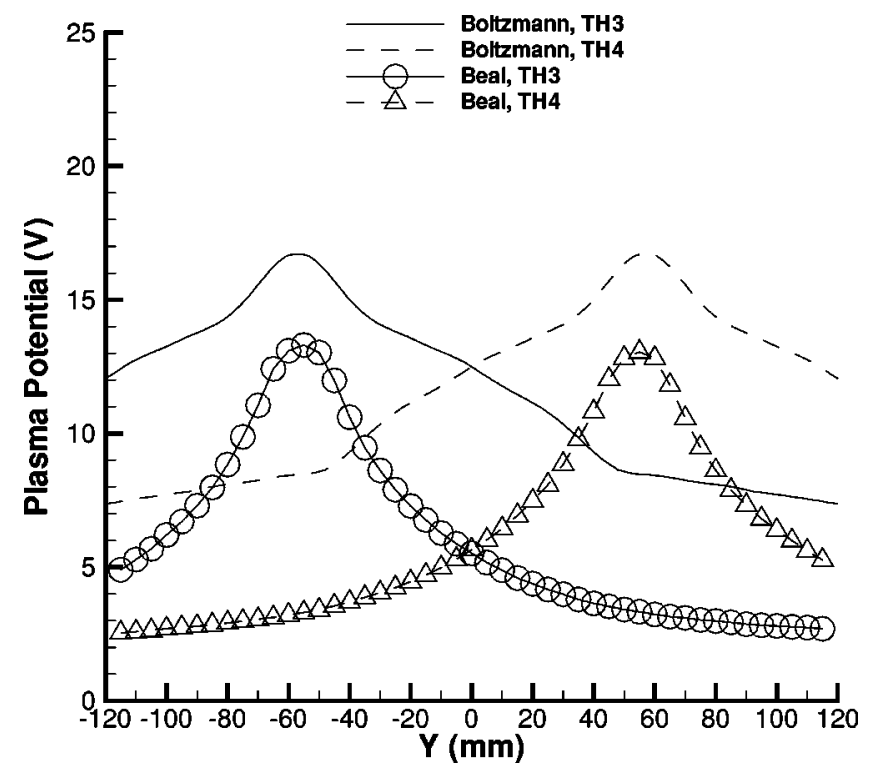

FIG. 10. Radial profiles of plasma potential at an axial distance of $100 \mathrm{~mm}$ from the thruster.

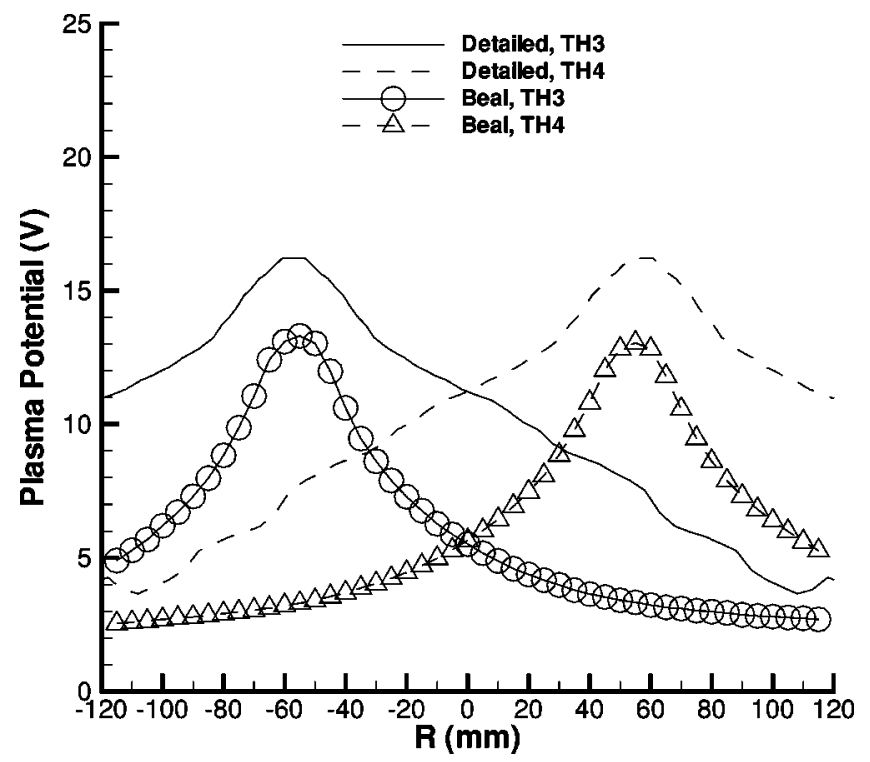

FIG. 11. Radial profiles of plasma potential at an axial distance of $100 \mathrm{~mm}$ from the thruster.

reasonable agreement with the measurements in terms of the peak temperatures, the radial gradients predicted by the model are significantly smaller than the measured data indicate.

The second set of data obtained from the Langmuir probe is electron density and this is the property that has greatest experimental uncertainty. ${ }^{2}$ The profiles of electron number density along the plume axis are shown in Fig. 16. Three different profiles of measured data are shown representing two different corrections to the raw data. ${ }^{2}$ The profile labeled "sheath" assumes that the probe collection radius is increased by a sheath of five Debye lengths, thus leading to a reduction in plasma density. The profile labeled "Laframboise" incorporates corrections due to the slightly different voltages applied to each of the three probes in the instrument. ${ }^{15}$ The correction is sensitive to the ratio of elec-

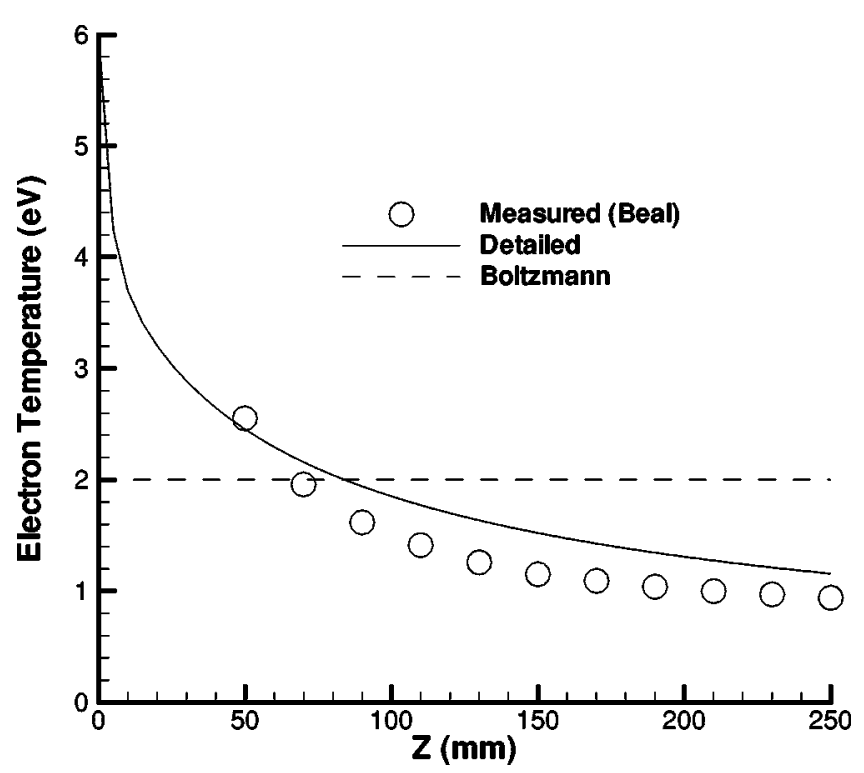

FIG. 12. Axial profiles of electron temperature along the plume centerline. 


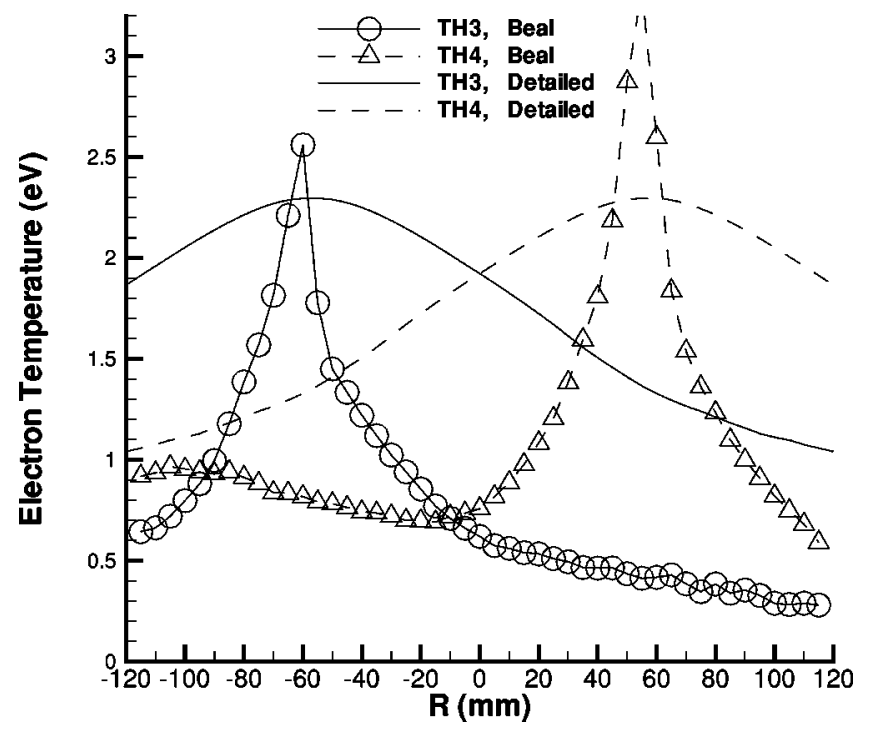

FIG. 13. Radial profiles of electron temperature at an axial distance of 50 $\mathrm{mm}$ from the thruster.

tron to ion temperature, and a ratio of one is assumed in the corrected data shown here. This correction also leads to a reduction in the measured number density. The comparisons indicate that both the Boltzmann nor the Detailed models predict near field densities that are significantly lower than any of the data derived from the experiments. However, beyond $200 \mathrm{~mm}$ from the thruster exit, both simulations are in reasonable agreement with the measured data. Additional simulations performed with a reduced ion temperature at the thruster exit is able to significantly increase the plasma density in the near field. However, the uncertainty in the plasma density measurements makes it inappropriate to draw any strong conclusions from such comparisons.

Figures 17 and 18 show radial profiles of electron number density at axial distances of 50 and $250 \mathrm{~mm}$ from the thruster, respectively. Due to the large amount of data in-

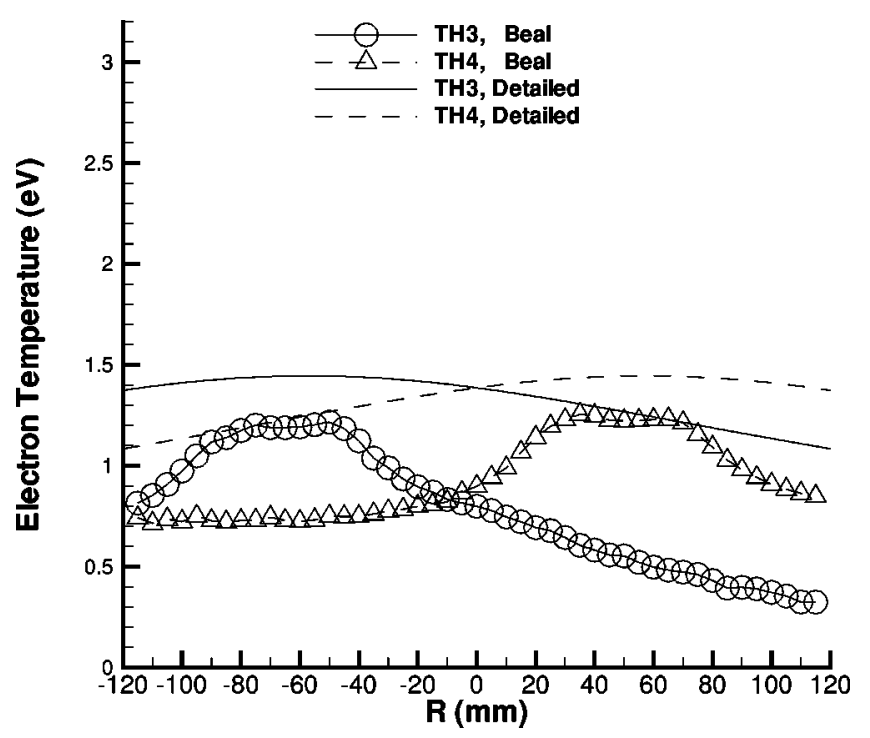

FIG. 14. Radial profiles of electron temperature at an axial distance of 150 $\mathrm{mm}$ from the thruster.

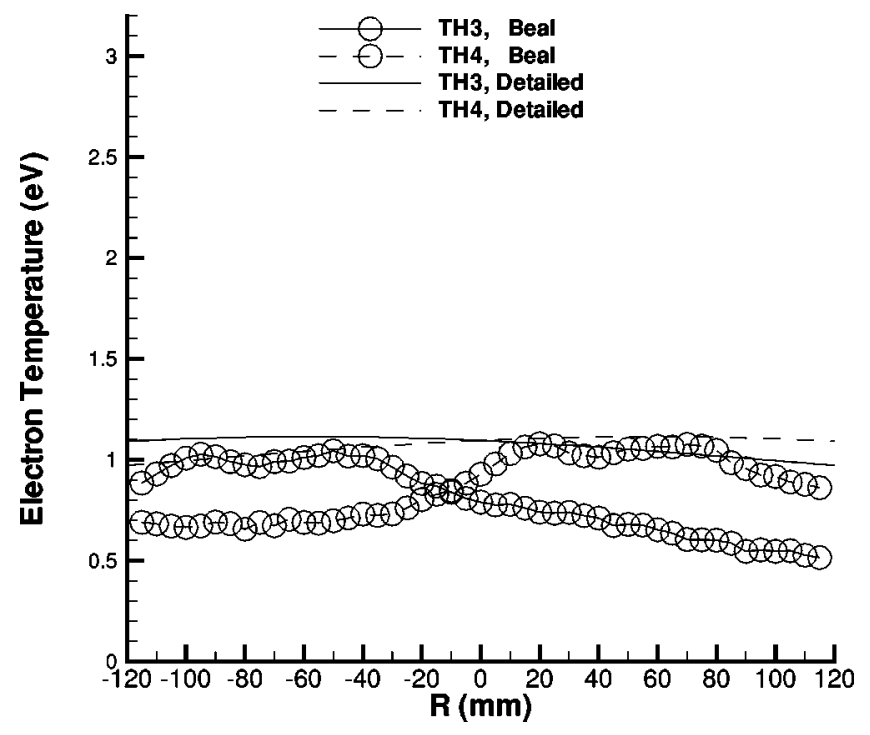

FIG. 15. Radial profiles of electron temperature at an axial distance of 250 $\mathrm{mm}$ from the thruster.

cluded, the profiles from only one thruster are displayed. In Fig. 17, in the near field, the simulations under predict the measured values over the entire radial profile. However, further from the thruster, as shown in Fig. 18, the simulations show quite good agreement with the corrected measurements.

Finally, the simulation results are compared with LIF measurements of $\mathrm{Xe}^{+}$velocity components obtained by Hargus and Charles ${ }^{5}$ in the near field plume. In Ref. 5, it is explained that the reported velocity data represent the peaks of the ion velocity distribution functions detected by the LIF diagnostic. Therefore, for consistency, in the simulations, the ion velocity distribution function is calculated throughout the flow field, and the peak of the distributions obtained. In several locations, particularly for the radial velocity component, two strong peaks are found in both the measured and simulated data.

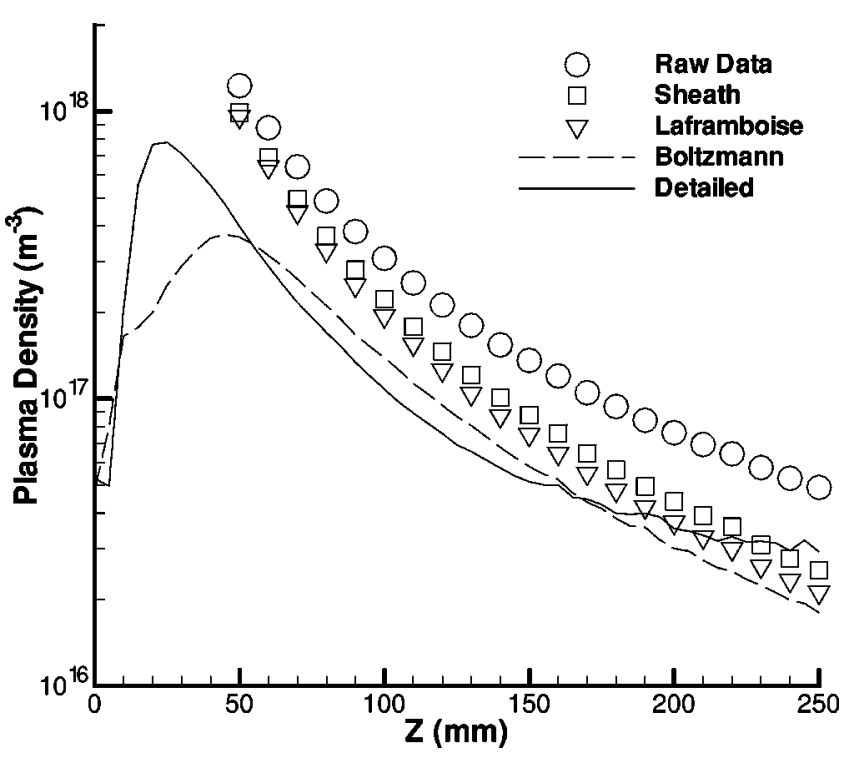

FIG. 16. Axial profiles of electron density along the plume centerline. 


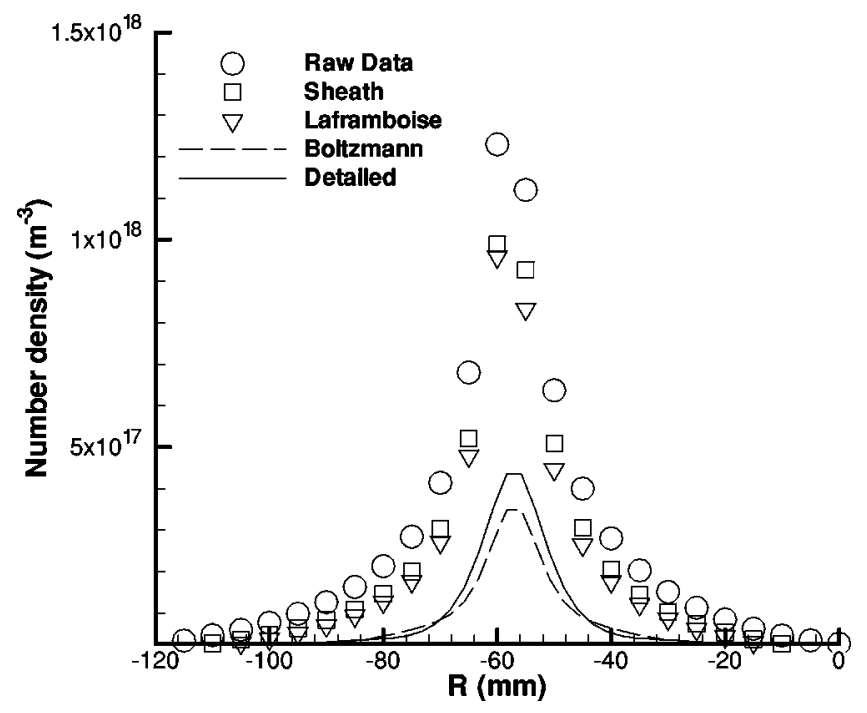

FIG. 17. Radial profiles of electron density at an axial distance of $50 \mathrm{~mm}$ from the thruster.

Figure 19 shows the profiles of ion velocity components along the plume centerline. Let us first consider the axial velocity component. Note that the thruster employs a small cone on the thruster face at the plume axis to provide protection against ion sputtering. The cone only protrudes $7 \mathrm{~mm}$ from the thruster face and could not easily be included in the simulation and so is omitted. This explains the small negative axial velocity components predicted by the simulations for $Z<10 \mathrm{~mm}$ that are absent from the measurements. The profile from the Boltzmann model rises instantaneously to the value assumed at the thruster exit of $17.7 \mathrm{~km} / \mathrm{s}$ that is higher than the measured value even at $80 \mathrm{~mm}$ from the thruster. By comparison, the Detailed model shows an extended acceleration region in which the computed velocity rises to exactly match the measured data at about $50 \mathrm{~mm}$ from the thruster. In terms of the radial velocity profiles, one can clearly see the two peaks found in the data sets repre-

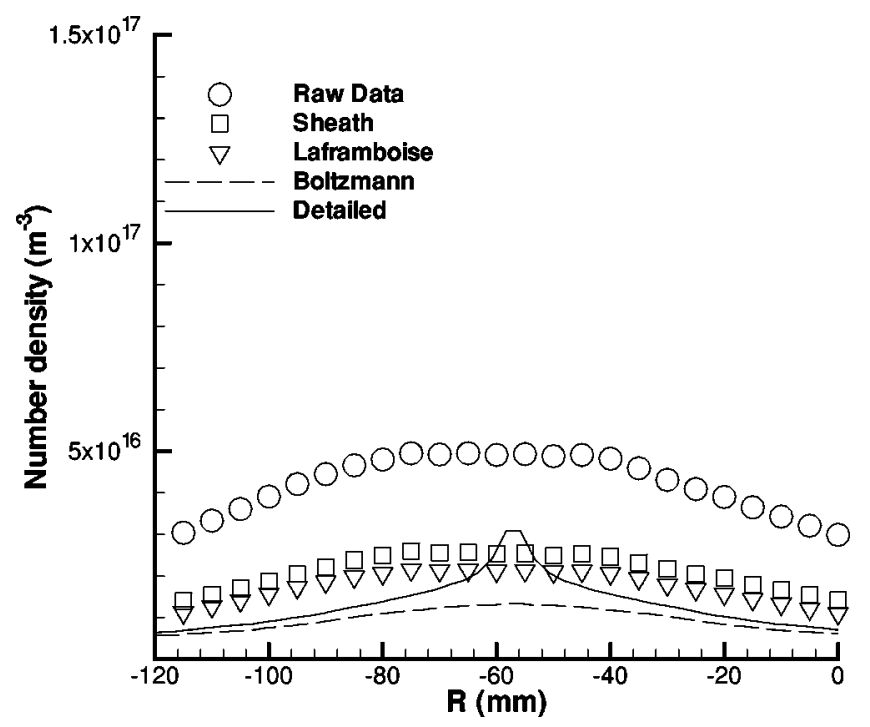

FIG. 18. Radial profiles of electron density at an axial distance of $250 \mathrm{~mm}$ from the thruster.

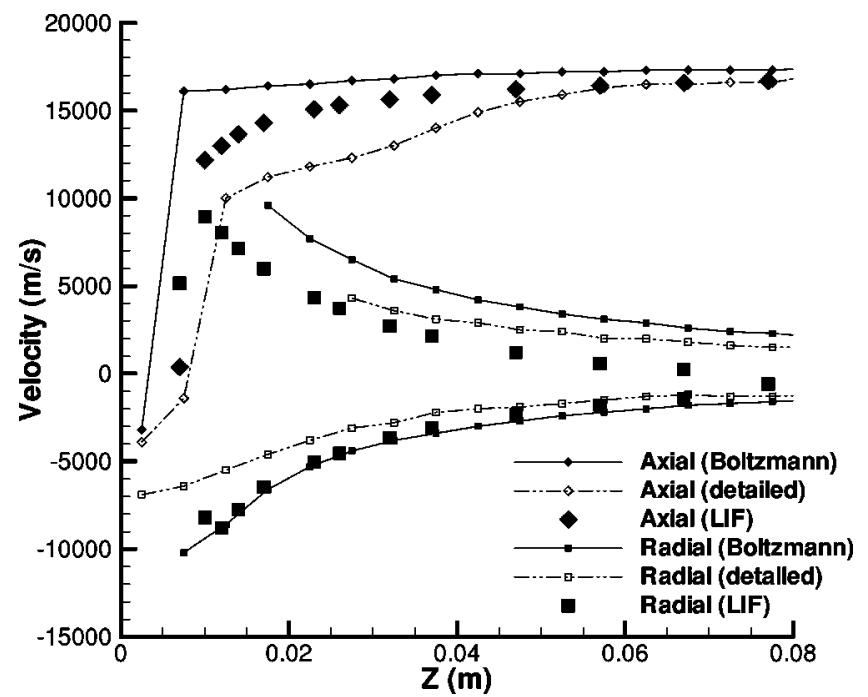

FIG. 19. Axial profiles of ion velocity components along the plume centerline.

senting populations of upward and downward moving ions. In each case, the Detailed model provides a slightly better correspondence to the measured data with the Boltzmann model tending to over predict the velocity magnitudes.

Figures 20 and 21 show radial profiles of ion velocity components at distances of 0 and $107 \mathrm{~mm}$ from the thruster. The axial velocity data in the thruster exit plane shown in Fig. 20 confirm the earlier statement that the ion velocity of $13.3 \mathrm{~km} / \mathrm{s}$ assumed at the thruster exit for the Detailed model is consistent with the LIF measurements. The velocity of $17.7 \mathrm{~km} / \mathrm{s}$ required in the Boltzmann model to obtain agreement with the far-field ion energy distribution function is too high. The measured radial velocity components in the thruster exit show a distinct asymmetry. Note that the center of the acceleration channel is at about $12 \mathrm{~mm}$ where the radial component is less than zero. This asymmetry has not been reproduced in the assumed thruster exit conditions for either simulation, but is expected to have only a small effect

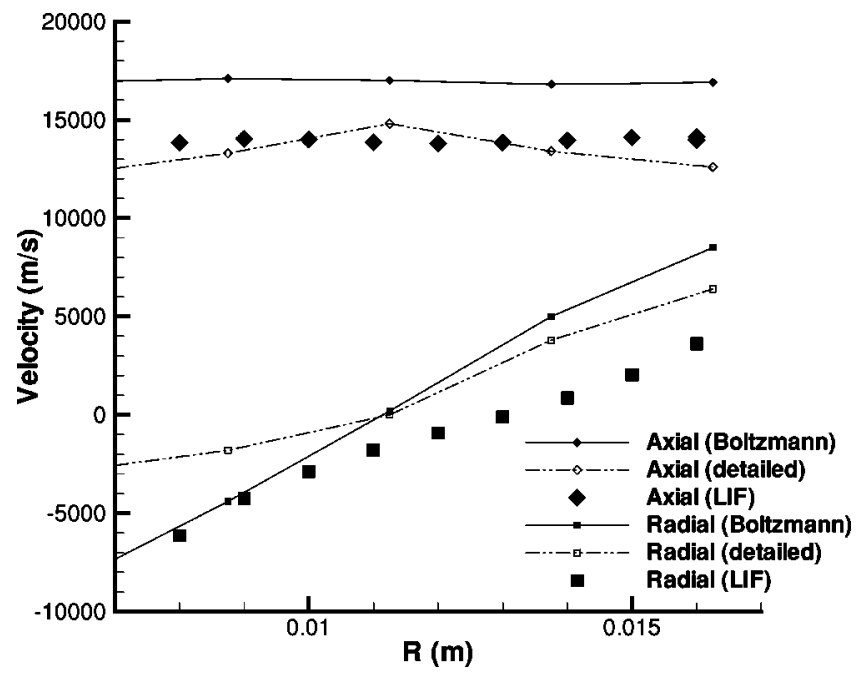

FIG. 20. Radial profiles of ion velocity components at an axial distance of 0 $\mathrm{mm}$ from the thruster. 


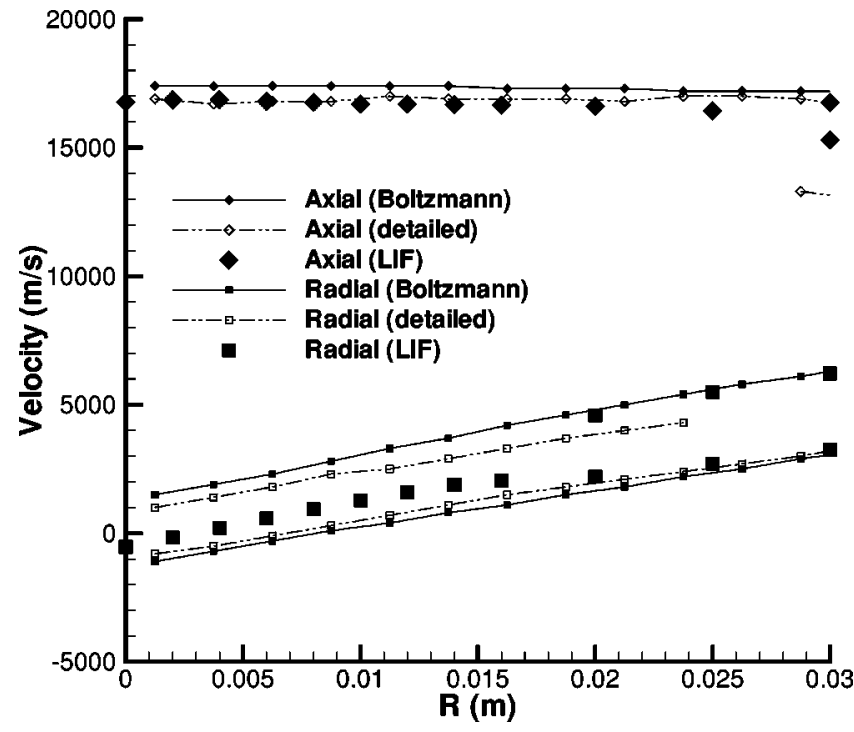

FIG. 21. Radial profiles of ion velocity components at an axial distance of $107 \mathrm{~mm}$ from the thruster.

on the overall flow field computations. The profiles at 107 $\mathrm{mm}$ from the thruster again show the Detailed model providing an accurate prediction of the axial component, with the Boltzmann model still at the assumed thruster exit value. in terms of the radial velocity components, the Detailed model tends to provide better prediction of the main peak of the distribution function.

\section{CONCLUSIONS}

A Detailed hybrid particle-fluid PIC-DSMC model was applied to model the plume flow from a small Hall thruster starting at the thruster exit and proceeding to the plume far field. The model included a detailed fluid description of the electrons based on their continuity, momentum, and energy equations. This model represents a significant increase in sophistication when compared to current Hall thruster plume models based on the Boltzmann relation. The Boltzmann and Detailed models were assessed by performing comparisons with a number of different data sets measured experimentally. The data sets comprised ion current density and ion energy distributions in the plume far field; in the plume near field, plasma potential was measured with a floating emissive probe, and both electron temperature and electron number density data were obtained using a triple Langmuir probe. Ion velocity components in the plume near field were also obtained using LIF. Both models were able to provide good agreement with the far field data, but in the case of the Boltzmann model, the measured ion energy distribution function could only be reproduced by assuming an ion velocity at the thruster exit that is much higher than the LIF and specific impulse data indicate. In comparison with the near field probe data, the Detailed model provided significant improvements over the Boltzmann model in terms of predicting the measured variations in plasma potential and electron temperature. For electron number density, there was considerable uncertainty in the measured values as indicated by two different correction schemes applied to the raw experimental data. The results from both models for electron number density were similar giving values significantly lowered than the measured data in the near field, and ultimately providing very good agreement with the experiments further from the thruster. The Detailed model accurately predicted the extended ion acceleration region outside the thruster that was indicated by the LIF data. By comparison, the Boltzmann model essentially indicated no significant ion acceleration outside the thruster. Both models predicted the multiple peaks in radial velocity component observed in the LIF data with the Detailed model providing slightly better correspondence to the measured data.

It is therefore concluded, with the possible exception of electron number density, that the Detailed model provides a significant improvement in the ability to simulate the nearfield processes in the plume of a Hall thruster. This may be important in assessing potential interactions between clusters of Hall thrusters, and for determining optical contamination from these devices. It is also a little disturbing that the established Boltzmann model could only provide results that agree with far field data by assuming unrealistic thruster exit conditions.

\section{ACKNOWLEDGMENTS}

The authors gratefully acknowledge the contributions to this work by Brian Beal and Alec Gallimore of the University of Michigan, and Bill Hargus of the Air Force Research Laboratory, for sharing their experimental data and for technical discussions in the course of this study. In addition, they wish to thank Bruce Pote of Busek, Co., for providing vital information on the BHT-200 Hall thruster.

${ }^{1}$ W. A. Hargus and G. Reed, AIAA 2002-3678, 38th AIAA/ASME/SAE/ ASEE Joint Propulsion Conference and Exhibit, Indianapolis, IN 2002.

${ }^{2}$ B. E. Beal, Doctoral thesis, University of Michigan, Ann Arbor, Michigan, 2003.

${ }^{3}$ B. E. Beal and A. D. Gallimore, IEPC 2003-35, 28th International Electric Propulsion Conference, Toulouse, France, 2003.

${ }^{4}$ B. E. Beal, A. D. Gallimore, and W. A. Hargus, AIAA 2003-5155, 39th AIAA/ASME/SAE/ASEE Joint Propulsion Conference and Exhibit, Huntsville, AL, 2003.

${ }^{5}$ W. A. Hargus and C. S. Charles, AIAA 2003-5154, 39th AIAA/ASME/ SAE/ASEE Joint Propulsion Conference and Exhibit, Huntsville, AL, 2003.

${ }^{6}$ G. A. Bird, Molecular Gas Dynamics and the Direct Simulation of Gas Flows (Oxford University Press, Oxford, 1994).

${ }^{7}$ C. K. Birdsall and A. B. Langdon, Plasma Physics Via Computer Simulation (Adam Hilger, London, 1991).

${ }^{8}$ M. W. Crofton and I. D. Boyd, AIAA 2003-4171, 39th AIAA/ASME/SAE/ ASEE Joint Propulsion Conference and Exhibit, Huntsville, AL, 2003.

${ }^{9}$ I. D. Boyd, J. Spacecr. Rockets 38, 381 (2001).

${ }^{10}$ M. Mitchner and C. H. Kruger, Partially Ionized Gases (Wiley, New York, 1973).

${ }^{11}$ E. Ahedo, P. Martinez-Cerezo, and M. Martinez-Sanchez, Phys. Plasmas 8, 3058 (2001).

${ }^{12}$ A. Dalgarno, M. R. C. McDowell, and A. Williams, Proc. R. Soc. London 250, 411 (1958).

${ }^{13}$ S. H. Pullins, Y. Chiu, D. J. Levandier, and R. A. Dressler, AIAA 20000603, 38th AIAA Aerospace Sciences Meeting, Reno, NV, 2000.

${ }^{14}$ S. Miller, D. J. Levandier, Y. Chiu, and R. A. Dressler, J. Appl. Phys. 91, 984 (2002).

${ }^{15}$ J. Laframboise, Rarefied Gas Dynamics, edited by J. H. deLeeuw, (Academic, New York, 1966), Vol. 2. 